\title{
An analysis of surgical decisions made during laparoscopic cholecystectomy in patients with incidental gallbladder cancer
}

\author{
Michael J. Horkoff, Julia Bowes, Francis R. Sutherland, Elijah Dixon, Chad Ball, Oliver F. Bathe \\ Division of General Surgery, University of Calgary
}

\section{INTRODUCTION:}

Prognosis for gallbladder cancer (GBC) is poor, and surgical resection remains the only modality to offer a chance of cure. ${ }^{1}$ Up to $30 \%$ of GBCs are discovered incidentally during histopathologic examination of gallbladders following cholecystectomy. ${ }^{2}$ Tumor seeding can occur if there is bile spillage during these cases. ${ }^{3}$ Thus, if GBC is suspected intraoperatively or preoperatively, specialist consultation is advised. We sought to identify if errors in surgical decision making were common in these cases.

OBJECTIVE:

To identify modifiable surgical decisions in cases of incidental gallbladder cancer where bile spillage may have been preventable.

\section{METHODS:}

We completed a retrospective analysis of patients that were treated for gallbladder cancer in Calgary between 2008 and 2012. We identified those patients in which gallbladder cancer was identified incidentally during a laparoscopic cholecystectomy or on pathology. We searched through their respective operative reports looking for events of bile spillage.
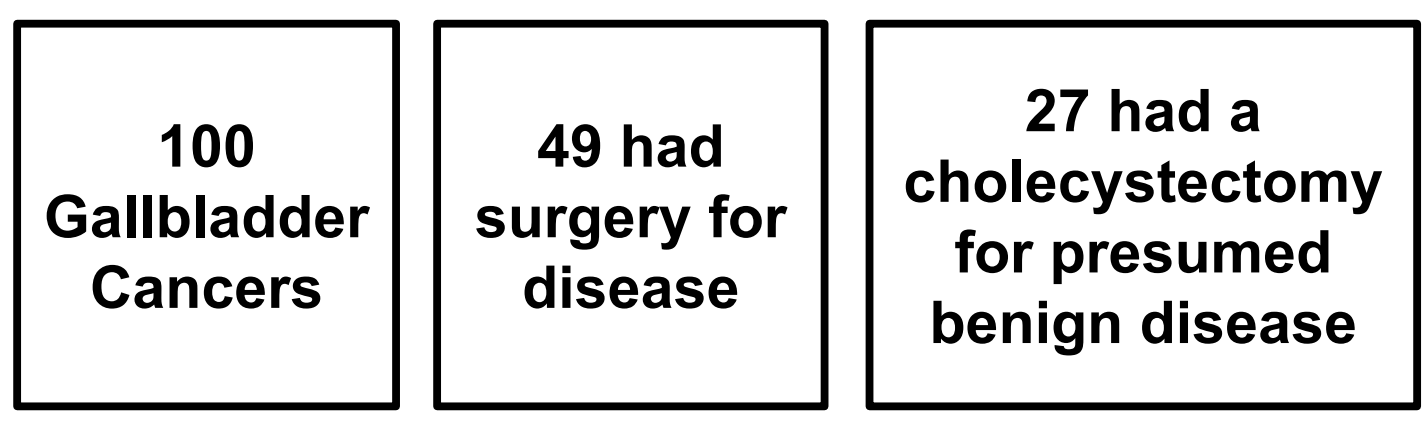

11 had bile spillage during the cholecystectomy

RESULTS:

INCIDENTAL GALLBLADDER CANCERS WITH BILE SPILLS DURING LAPAROSCOPIC CHOLECYSTECTOMY

\begin{tabular}{|c|c|c|c|c|c|}
\hline \multirow[b]{2}{*}{ Age } & \multirow{2}{*}{$\begin{array}{c}\text { Bile Spill } \\
\text { Intentional } \\
\text { or } \\
\text { Accidental }\end{array}$} & \multicolumn{2}{|c|}{ Preoperative Imaging } & \multirow[b]{2}{*}{$\begin{array}{l}\text { OR Report of } \\
\text { Malignancy }\end{array}$} & \multirow[b]{2}{*}{$\begin{array}{c}\mathbf{T} \\
\text { Stage }\end{array}$} \\
\hline & & Ultrasound & CT Scan & & \\
\hline 61 & Accidental & Calculi & N/A & None & $\mathrm{T} 3 \mathrm{Nx}$ \\
\hline 64 & Accidental & N/A & Cholecystitis & None & $\mathrm{T} 2 \mathrm{NI}$ \\
\hline 53 & Intentional & CBD Stone & N/A & None & $\mathrm{T} 3 \mathrm{Nx}$ \\
\hline 68 & Intentional & N/A & Cholecystitis & None & $\mathrm{T} 2 \mathrm{Nx}$ \\
\hline 62 & Intentional & Cholecystitis & $\mathrm{N} / \mathrm{A}$ & None & $\mathrm{T} 3 \mathrm{NI}$ \\
\hline 53 & Intentional & Cholecystitis & N/A & None & TIbNx \\
\hline 50 & Intentional & $\begin{array}{l}\text { **Possible } \\
\text { Malignancy }\end{array}$ & N/A & **Suspicious Lesion & $\mathrm{T} 3 \mathrm{Nx}$ \\
\hline 60 & Intentional & Calculi & N/A & **Suspicious Nodularity & $\mathrm{T} 2 \mathrm{Nx}$ \\
\hline 52 & Accidental & N/A & N/A & Conv to open & $\mathrm{T} 3 \mathrm{NI}$ \\
\hline 70 & Accidental & Calculi & N/A & **Suspicious Lesion & $\mathrm{T} 2 \mathrm{Nx}$ \\
\hline 85 & Intentional & CBD Stone & N/A & $\begin{array}{l}* * \text { Suspicious Lesion, } \\
\text { Conv to open }\end{array}$ & $\mathrm{T} 3 \mathrm{Nx}$ \\
\hline
\end{tabular}

\section{CONCLUSION:}

In summary, we identified 5 out of 27 cases of incidental GBC where different surgical decisions may have prevented a bile spill. Patients with bile spillage were less likely to have a radical resection performed and had a significantly shorter disease free survival. Surgeons performing cholecystectomies must be aware of the signs of gallbladder cancer and be prepared to alter the surgical plan based on a high index of suspicion.

References:

Groen, PC., Gores, GJ., LaRusso, NF., et al. Biliary tract cancers. NEJM. (1999). 341:1368-78.

2. Cho, SY., Park, SJ., Kim, SH., et al. Comparative analysis between clinical outcomes of primary radical resection and second completion radical resection for T2 gallbladder cancer: single-center experience. World J Surg. (2010). 34(7):1572-8.

3. Wibbenmeyer, LA., Wade, TP., Chen, RC., et al. Laparoscopic cholecystectomy can disseminate in situ carcinoma of the gallbladder. J Am Coll Surg. (1995). 181(6):504-10. 